\title{
$\beta$-asarone relieves chronic unpredictable mild stress induced depression by regulating the extracellular signal-regulated kinase signaling pathway
}

\author{
HAIYING DONG ${ }^{1 *}$, WEILIANG CONG ${ }^{2 *}$, XIWEN GUO ${ }^{3}$, YUHUA WANG $^{3}$, \\ SHENGJU TONG ${ }^{4}$, QIANG $\mathrm{LI}^{5}$ and CHENGCHONG $\mathrm{LI}^{3}$ \\ ${ }^{1}$ Institute of Pathology, Qiqihar Medical University, Qiqihar, Heilongjiang $161006 ;{ }^{2}$ Department of Anaesthesiology, \\ The Third Affiliated Hospital of Qiqihar Medical University, Qiqihar, Heilongjiang 161000; ${ }^{3}$ School of Mental Health, \\ Qiqihar Medical University, Qiqihar, Heilongjiang 161006; Departments of ${ }^{4}$ General Surgery and ${ }^{5}$ Cardiology, \\ The Third Affiliated Hospital of Qiqihar Medical University, Qiqihar, Heilongjiang 161000, P.R. China
}

Received March 6, 2018; Accepted June 29, 2018

DOI: $10.3892 /$ etm.2019.8018

\begin{abstract}
The present study aimed to investigate the effect of $\beta$-asarone treatment in a rat model of depression induced by chronic unpredictable mild stress (CUMS) and to further explore the underlying molecular mechanisms. A rat model of depression was established by subjecting rat to CUMS and treated with various concentrations of $\beta$-asarone (12.5, 25 and $50 \mathrm{mg} / \mathrm{kg} /$ day) and fluoxetine (20 mg/kg/day). Next, behavioral tests, including an open field, sucrose preference and forced swimming tests, were performed. In addition, the apoptosis of hippocampal neuronal cells was determined by flow cytometry, gene expression levels were detected by reverse transcription-quantitative polymerase chain reaction and protein levels were determined by western blot assay. The results revealed that $\beta$-asarone significantly mitigated CUMS-induced depression-like behavior, evidenced by the increased sucrose intake, crossing and rearing numbers, and decreased immobility time in the forced swimming test. Furthermore, $\beta$-asarone significantly decreased the apoptosis rate of hippocampal neuronal cells in rats subjected to CUMS. $\beta$-asarone was also found to enhance CREB, BDNF, Trk-B and Bcl-2 levels, and reduce Bad level in the hippocampus of CUMS-treated rats. In addition, the activation of extracellular signal-regulated kinase pathway inhibited by CUMS was promoted by $\beta$-asarone treatment. In conclusion, the present
\end{abstract}

Correspondence to: Mr. Chengchong Li, School of Mental Health, Qiqihar Medical University, 333 Bukui Street, Jianhua, Qiqihar, Heilongjiang 161006, P.R. China

E-mail: licc201803@163.com

*Contributed equally

Key words: depression, chronic unpredictable mild stress, $\beta$-asarone, extracellular signal-regulated kinase pathway study findings indicated the antidepressant-like effects of $\beta$-asarone on CUMS-induced depression in rats.

\section{Introduction}

As a common neuropsychiatric disorder, depression is one of the major causes of disability worldwide (1). Depression is characterized by a variety of symptoms, including persistent low mood, cognitive impairment, intellectual ability retardation and somatic symptoms (2). With the rapid social and economic development, stress and adversity are becoming more severe, which may lead to depression. According to the prediction of WHO, the incidence of depression increases $113 \%$ annually and is expected to become the second largest disease burden worldwide by the end of 2020 (3). Although the cause of depression is poorly understood, stress is considered to be the main contributing factor leading to biochemical alterations in the brain that present as the clinical symptoms of depression $(4,5)$. To date, $>20$ types of animal models of depression have been established, and the chronic unpredictable mild stress (CUMS) model, originally developed by Willner et al (6), is the most widely used model. At present, tricyclic antidepressants, monoamine oxidase inhibitors and selective 5-hydroxytryptamine reuptake inhibitors are the most commonly used drugs for the treatment of depression; however, their effect is not satisfactory. Therefore, identifying drugs with high efficiency and low toxicity for the treatment of depression is currently urgent.

$\beta$-asarone (also known as cis-2,4,5-trimethoxy-1-allyl phenyl) is the main active ingredient of the traditional Chinese medicinal herb Acorus tatarinowii Schott. $\beta$-asarone has various pharmacological properties, and can easily pass through the blood-brain barrier and be distributed in the brain (7). Recent data have demonstrated that $\beta$-asarone can significantly affect the central nervous system and serves an important role in relieving neuronal apoptosis (8-10). Studies have also evaluated the anti-tumor effect of $\beta$-asarone $(11,12)$. Furthermore, it has been reported that $\beta$-asarone has antidepressive effects (13-17); however, the precise role and underlying mechanisms of these effects remain largely unclear. 
Therefore, the current study aimed to investigate the effects of $\beta$-asarone administration in a rat model of depression induced by CUMS and to further explore the underlying molecular mechanisms.

\section{Materials and methods}

Depression model establishment. In total, 120 healthy 6-week-old male Sprague-Dawley rats (180-210 g) were obtained from the Vital River Laboratory Animal Technology Co., Ltd. (Beijing, China). The rats were fed under standard conduction (12-h light/dark cycle; $55 \pm 5 \%$ humidity; $22 \pm 2^{\circ} \mathrm{C}$ ), and were provided with food and water ad libitum. All animal experiments were conducted according to the Guidelines for the Care and Use of Laboratory Animals by the National Institutes of Health. The present study was approved by the Ethics Committee of Qiqihar Medical University (Qiqihar, China).

The rat model of depression was established by CUMS treatment for 6 weeks as described in a previous study (18). Briefly, rats were group-housed and allowed to adapt to the environment for 1 week. Next, rats were single-housed and subjected to a variety of mild stressors for 6 weeks, with the exception of the control group rats, which were undisturbed in their cages in a separated room throughout the following 6 weeks. The mild stressors were as follows: Food deprivation for $24 \mathrm{~h}$; water deprivation for $24 \mathrm{~h}$; overnight illumination; cage tilt $\left(45^{\circ}\right)$ for $7 \mathrm{~h}$; soiled cage $(200 \mathrm{ml}$ water in $100 \mathrm{~g}$ sawdust bedding); foreign object exposure; light/dark perversion; hanging the rat on a balance bar with rope for $10 \mathrm{~min}$; physical restraint for $3 \mathrm{~h}$; 1 -min tail pinch $(1 \mathrm{~cm}$ from the beginning of the tail); 5-min oscillation; and exposure to white noise for $1 \mathrm{~h}$. To ensure the unpredictability of the experiment, all stressors were performed randomly. Two or three types of stimuli were randomly scheduled daily for a total of 28 days. The same stimulus was not repeated for 3 consecutive days.

Rats were randomly divided into six groups $(n=20$ per group), as follows: i) Control group, unstressed + saline vehicle ( $0.01 \mathrm{ml} / \mathrm{g}$ body weight); ii) model group, CUMS + saline vehicle ( $0.01 \mathrm{ml} / \mathrm{g}$ body weight); iii) CUMS $+12.5 \mathrm{mg} / \mathrm{kg} / \mathrm{day} \beta$-asarone treatment group; iv) CUMS $+25 \mathrm{mg} / \mathrm{kg} /$ day $\beta$-asarone treatment group; v) CUMS $+50 \mathrm{mg} / \mathrm{kg} /$ day $\beta$-asarone treatment group; and vi) CUMS $+20 \mathrm{mg} / \mathrm{kg} /$ day fluoxetine treatment group, serving as the positive control. Beginning on week 4 , the rats were orally administered with $\beta$-asarone or fluoxetine every day for 3 weeks. $\beta$-asarone and fluoxetine hydrochloride were obtained from Hubei Bangsun Chemical Co., Ltd. (Wuhan, China). At the end of the treatment, behavioral tests were conducted, and the hippocampal tissues of the rats were collected (week 6 after CUMS) according to the previous study $(18,19)$. Rats were decapitated after anesthetization with $5 \%$ chloral hydrate $(350 \mathrm{mg} / \mathrm{kg}$; intraperitoneally). No rats exhibited signs of peritonitis following the administration of anesthetic.

Open field test. The open field test was performed as previously described (20). The open field device used was a four-sided black metallic enclosure (100x100x40 cm) with a white open floor and was divided into 25 equal sectors by red lines. Each rat was placed alone in the center of the arena and was allowed to explore freely for $5 \mathrm{~min}$. The frequency of rearing (rat erected on its hind legs) and number of crossings (rat entered into a new sector with four paws) were recorded.

Forced swimming test. The forced swimming test was performed according to a previously described method, with minor modifications (21). Briefly, each rat was forced to swim in a cylindrical glass container (height, $50 \mathrm{~cm}$; diameter, $20 \mathrm{~cm})$ with $30 \mathrm{~cm}$ of water $\left(22 \pm 1^{\circ} \mathrm{C}\right)$ for $6 \mathrm{~min}$. In the last 4 min, the immobile time (rat floating in the water without swimming) was recorded.

Sucrose preference test. The sucrose preference test was performed every week [from the beginning of the experiment (week 1) to week 6 (after the last treatment)], as described previously (22). The sucrose preference ratio was calculated as follows: Sucrose preference value $(\%)=$ Sucrose intake $(\mathrm{g}) \mathrm{x}$ $100 \% /[$ sucrose intake $(\mathrm{g})+$ water intake $(\mathrm{g})]$.

Detection of the apoptosis in the hippocampus. Extraction of hippocampal tissue from rats of different groups and single cell suspension preparation were performed according to a previous study (23). Briefly, hippocampal tissue was extracted on ice, washed with cold saline, and then a 200 mesh sieve was used to mechanically dissociate the tissues into a single cell suspension. Subsequently, the cell suspension was centrifuged at $4^{\circ} \mathrm{C}$ for $5 \mathrm{~min}$ at $670.8 \mathrm{xg}$, and the supernatant was discarded. Finally, to detect the apoptosis in the hippocampus of rats, an Annexin V-FITC Apoptosis Detection kit (Cell Signaling Technology, Inc., Danvers, MA, USA) was used as per the manufacturer's protocol. Flow cytometry (BD Biosciences, Franklin Lakes, NJ, USA) was then used to analyze cell apoptosis. Tests were repeated three times.

Reverse transcription-quantitative polymerase chain reaction $(q P C R)$. To extract the total RNA from hippocampal tissues, TRIzol reagent (Invitrogen; Thermo Fisher Scientific, Inc., Waltham, MA, USA) was used in line with the manufacturer's protocol. Total RNA concentration was detected using Nanodrop2000 (Thermo Fisher Scientific, Inc.). cDNA was then generated using the TaqMan microRNA Reverse Transcription kit (Thermo Fisher Scientific, Inc.), following the manufacturer's protocol. Subsequently, the TaqMan ${ }^{\circledR}$ Universal PCR Master Mix kit (Thermo Fisher Scientific, Inc.) was utilized to examine the gene expression. The qPCR amplification conditions were as follows: $95^{\circ} \mathrm{C}$ for $10 \mathrm{~min}$, followed by 37 cycles of $95^{\circ} \mathrm{C}$ for $10 \mathrm{sec}$ and $60^{\circ} \mathrm{C}$ for $60 \mathrm{sec}$. The primer sequences were obtained as required, and were as follows: cAMP response element binding protein (CREB) forward, 5'-ACAGATTGCCACATTAGC-3', and reverse, 5'-CTCCTCCCTGGGTAATGG-3'; brain-derived neurotrophic factor (BDNF) forward, 5'-CCCTTCTACACTTTA CCTCTTG-3', and reverse, 5'-GTTTCACCCTTTCCACTC CTA-3'; tropomyosin receptor kinase B (Trk-B) forward, 5'-GGGGCTTATGCTTGCTGGTC-3', and reverse, 5'-CTC TGGGTCAATGCTGTTAGGTT-3'; B-cell lymphoma-2 (Bcl-2) forward, 5'-GGGACGCGA AGTGCTATTGG TA-3', and reverse, 5'-CAGGCTGGAAGGAGAAGATGC-3'; Bcl-2-associated death promoter (Bad) forward, 5'-ACACGC CCTAGGCTTGAGGA-3', and reverse, 5'-GGCTCAAAC 
TCTGGGATCTGGA-3'; and GAPDH forward, 5'-GACAAC TTTGGCATCGTGGA-3', and reverse, 5'-ATGCAGGGA TGATGTTCTGG-3'. The $2^{-\Delta \Delta C q}$ method was applied for relative gene expression quantification (24).

Western blot analysis. To measure the protein levels of CREB, BDNF, Trk-B, Bcl-2, Bad, extracellular signal-regulated kinase (ERK) and phosphorylated (p)-ERK in the hippocampus of rats from different groups, western blot analysis was conducted. Total proteins from the hippocampus of rats were extracted using a radioimmunoprecipitation assay buffer (Beijing Solarbio Science \& TechnologyCo.,Ltd.,Beijing,China).The protein quantification was calculated using the BCA assay (Thermo Fisher Scientific, Inc.), according to the manufacturer's protocol. An equal amount of protein samples ( $25 \mu \mathrm{g} / \mathrm{lane})$ was resolved by $10 \%$ SDS-PAGE and then blotted onto polyvinylidene difluoride membranes. The membranes were first blocked with 5\% skim milk at room temperature for $2 \mathrm{~h}$, and then incubated with a primary antibody against CREB (cat. no. 9197; 1:1,000; Cell Signaling Technology, Inc.), BDNF (cat no. Ab108319; 1:1,000; Abcam), Trk-B (cat. no. 4607; 1:1,000; Cell Signaling Technology, Inc.), Bcl-2 (cat. no. 4223; 1:1,000; Cell Signaling Technology, Inc.), Bad (cat. no. 9239; 1:1,000; Cell Signaling Technology, Inc.), ERK (cat. no. 4695; 1:1,000; Cell Signaling Technology, Inc.), p-ERK (cat. no. 4370; 1:1,000; Cell Signaling Technology, Inc.) or $\beta$-actin (cat. no. 4970; 1:1,000; Cell Signaling Technology, Inc.) at $4^{\circ} \mathrm{C}$ overnight. Subsequently, the membranes were incubated with horseradish peroxidase-conjugated anti-rabbit IgG secondary antibodies (cat. no. 7074; 1:2,000; Cell Signaling Technology, Inc.) at room temperature for $4 \mathrm{~h}$. To visualize the protein blots, an enhanced chemiluminescence detection kit (Cell Signaling Technology, Inc.) was used in accordance with the manufacturer's protocol. ImageJ 1.38X (National Institutes of Health, Bethesda, MD, USA) was used to perform densitometry.

Statistical analysis. All experiments were repeated three times. Data are displayed as the mean \pm standard deviation. SPSS version 17.0 software (SPSS, Inc., Chicago, IL, USA) was used for data analysis. Student's t-test or one-way analysis of variance followed by Tukey's test was used to assess comparisons between groups. $\mathrm{P}<0.05$ was considered to denote a statistically significant difference.

\section{Results}

$\beta$-asarone ameliorates body weight reduction induced by CUMS. As shown in Fig. 1, at the beginning of the test, the body weight of rats in different groups was similar. After rats were subjected to CUMS for 2 weeks, their body weight was significantly decreased in comparison with that in the control group $(\mathrm{P}<0.05)$. Treatment with different concentrations of $\beta$-asarone $(12.5,25$ and $50 \mathrm{mg} / \mathrm{kg} / \mathrm{day})$ and with $20 \mathrm{mg} / \mathrm{kg} / \mathrm{day}$ fluoxetine significantly ameliorated the reduction in the body weight that was induced by CUMS. $\beta$-asarone administered at $50 \mathrm{mg} / \mathrm{kg} /$ day exhibited the most marked effect on the body weight of CUMS induced rats.

$\beta$-asarone ameliorates depression-associated symptoms induced by CUMS. To investigate the effects of $\beta$-asarone on CUMS-induced depression-like behavior, the sucrose

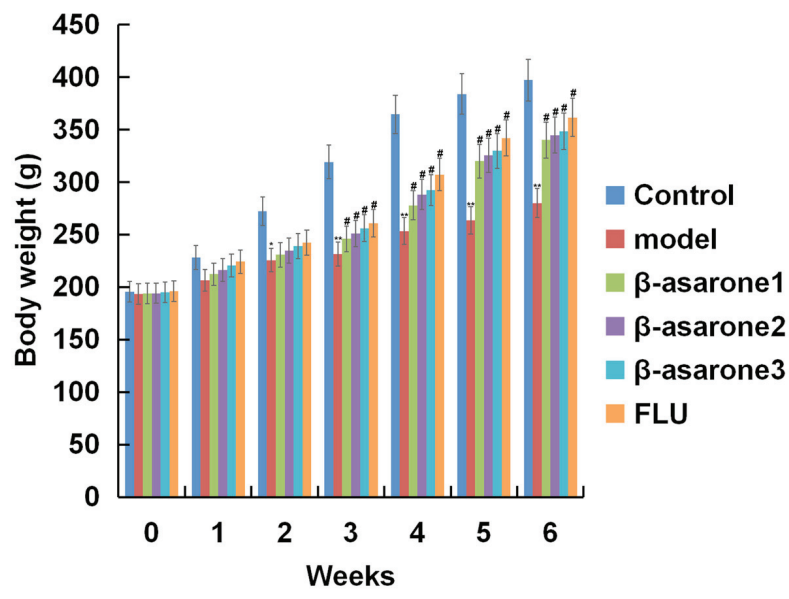

Figure 1. Effect of $\beta$-asarone on the body weight of rats. Data are expressed as the mean \pm standard deviation. ${ }^{*} \mathrm{P}<0.05$ and ${ }^{* *} \mathrm{P}<0.01$ vs. control group; ${ }^{\#} \mathrm{P}<0.05$ vs. model group. $\beta$-asarone $1 / 2 / 3$, depression model rats treated with $12.5,25$ or $50 \mathrm{mg} / \mathrm{kg} \beta$-asarone, respectively; FLU, fluoxetine-treated depression rats.

preference, forced swimming and open field tests were conducted in the present study. As shown in Fig. 2A, a significant decrease in sucrose intake was observed in the model group when compared with the control group, and $(12.5,25$ and $50 \mathrm{mg} / \mathrm{kg} / \mathrm{day}$ ) $\beta$-asarone or $20 \mathrm{mg} / \mathrm{kg} /$ day fluoxetine treatment significantly increased the sucrose intake. In addition, the immobility time in the forced swimming test was significantly enhanced in rats of the model group, while $(12.5,25$, $50 \mathrm{mg} / \mathrm{kg} / \mathrm{day}$ ) $\beta$-asarone or $20 \mathrm{mg} / \mathrm{kg} /$ day fluoxetine treatment notably reduced this enhancement (Fig. 2B). Furthermore, the locomotor activity was detected in the open field test, and the results are shown in Fig. 2C and D. Compared with the control group, the crossing and rearing number in the model rats was significantly decreased, while treatment with $(12.5,25$ and $50 \mathrm{mg} / \mathrm{kg} / \mathrm{day}) \beta$-asarone or $20 \mathrm{mg} / \mathrm{kg} /$ day fluoxetine significantly eliminated these changes. Furthermore, $50 \mathrm{mg} / \mathrm{kg} / \mathrm{day}$ $\beta$-asarone exhibited the most marked effect on CUMS induced depression-associated symptoms in rats. The findings indicated that the $\beta$-asarone and fluoxetine treatments significantly relieved CUMS-induced depression.

$\beta$-asarone prevents CUMS-induced apoptosis in the hippocampus. The findings demonstrated that, compared with the control group, the apoptosis rate of hippocampal neuronal cells significantly increased in rats of the model group. By contrast, $(12.5,25$ and $50 \mathrm{mg} / \mathrm{kg} /$ day $) \beta$-asarone and $20 \mathrm{mg} / \mathrm{kg} /$ day fluoxetine treatment significantly decreased the apoptosis rate in hippocampal cells as compared with that observed in the model group (Fig. 3). $\beta$-asarone administered at $50 \mathrm{mg} / \mathrm{kg} / \mathrm{day}$ exhibited the most marked effect on CUMS-induced apoptosis in the hippocampus of rats.

$\beta$-asarone enhances CREB, BDNF and Trk-B levels in the hippocampus of CUMS-treated rats. The effect of $\beta$-asarone on the levels of CREB, BDNF and Trk-B in the hippocampus of rats subjected to CUMS was determined. As shown in Fig. 4, compared with the control group, the mRNA and protein levels of CREB, BDNF and Trk-B were significantly decreased in the hippocampus of rats in the model group; however, $(12.5,25$ and 
A

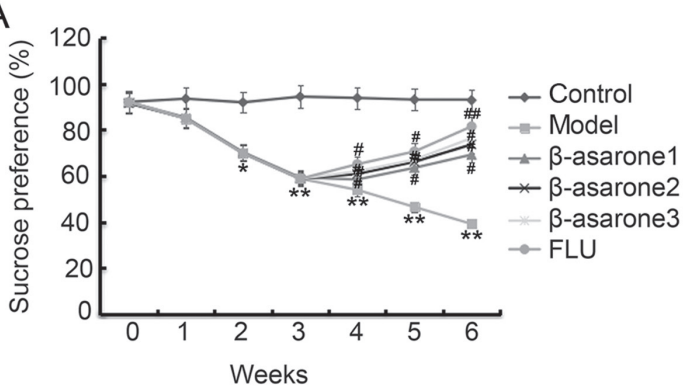

C

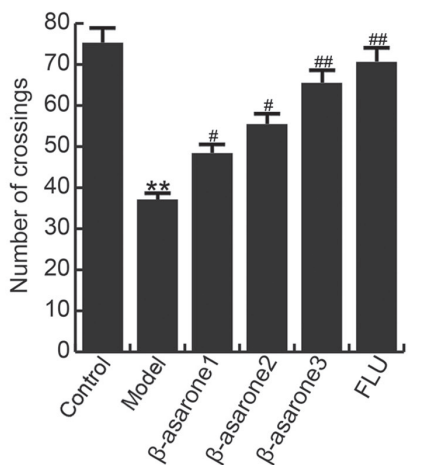

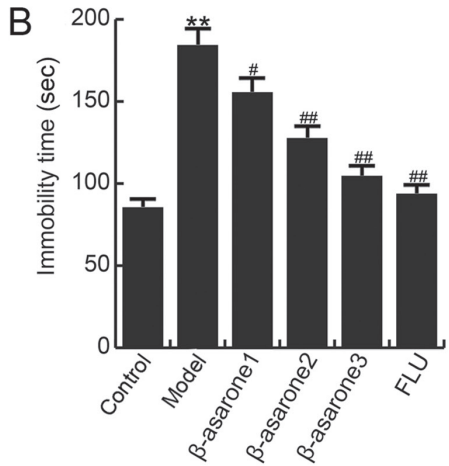

D

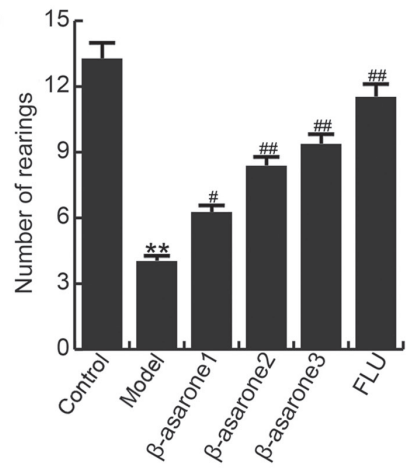

Figure 2. Effect of $\beta$-asarone on chronic unpredictable mild stress-induced depression-like behavior. (A) Sucrose preference test results, (B) forced swimming test results, and the number of $(C)$ crossings and (D) rearings in the open field test are shown. Data are expressed as the mean \pm standard deviation. ${ }^{*} \mathrm{P}<0.05$ and ${ }^{* *} \mathrm{P}<0.01$, vs. control group; ${ }^{\#} \mathrm{P}<0.05$ and ${ }^{\# \#} \mathrm{P}<0.01$, vs. model group. $\beta$-asarone1/2/3, depression model rats treated with $12.5,25 \mathrm{or} 50 \mathrm{mg} / \mathrm{kg} \beta$-asarone, respectively; FLU, fluoxetine-treated depression rats.
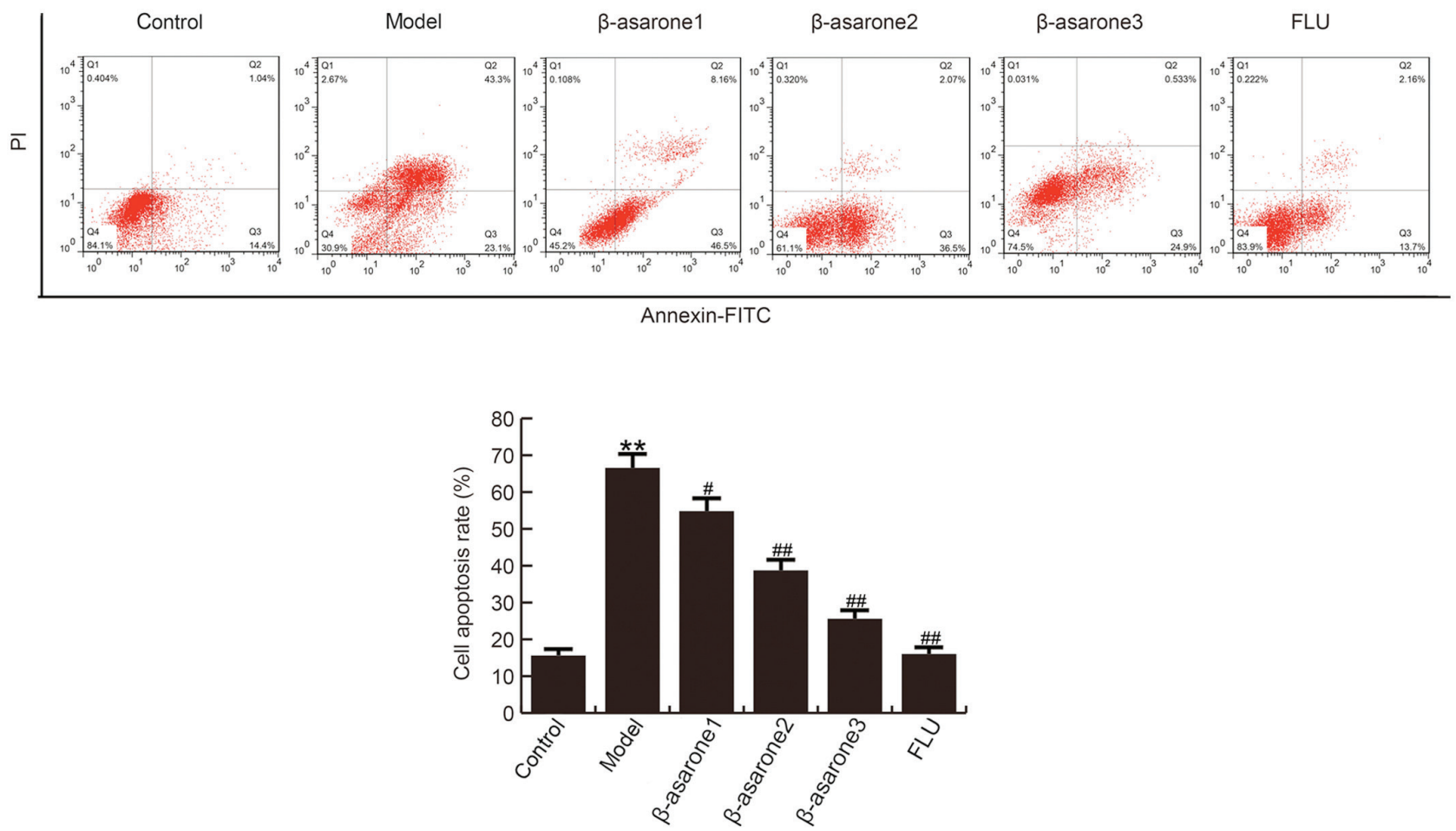

Figure 3. $\beta$-asarone prevents apoptosis in the hippocampus induced by chronic unpredictable mild stress. Hippocampal cells obtained from the rats in the different groups were stained with Annexin V-FITC and propidium iodide, and the labeling was analyzed using flow cytometry. Data are expressed as the mean \pm standard deviation. ${ }^{* *} \mathrm{P}<0.01$ vs. control group; ${ }^{*} \mathrm{P}<0.05$ and ${ }^{\# \#} \mathrm{P}<0.01$, vs. model group. $\beta$-asarone1/2/3, depression model rats treated with $12.5,25$ or $50 \mathrm{mg} / \mathrm{kg} \beta$-asarone, respectively; FLU, fluoxetine-treated depression rats.

$50 \mathrm{mg} / \mathrm{kg} / \mathrm{day}) \beta$-asarone and $20 \mathrm{mg} / \mathrm{kg} /$ day fluoxetine treatment significantly inhibited this reduction. $\beta$-asarone administered at
$50 \mathrm{mg} / \mathrm{kg} /$ day exhibited the most marked effect on CREB, BDNF and Trk-B levels in the hippocampus of CUMS-treated rats. 
A

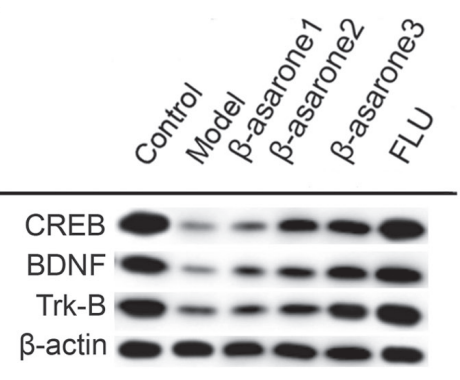

$B$

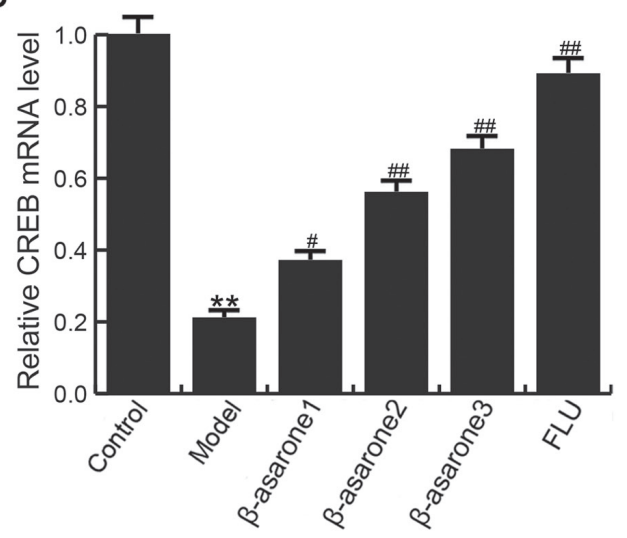

D

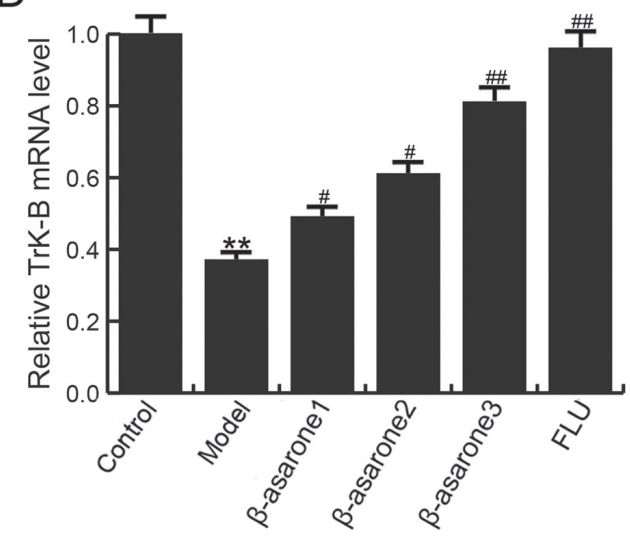

Figure 4. $\beta$-asarone increases CREB, BDNF and Trk-B levels in the hippocampus of chronic unpredictable mild stress-treated rats. (A) Western blot analysis to examine CREB, BDNF and Trk-B protein levels. (B) CREB, (C) BDNF and (D) Trk-B mRNA levels in different groups were detected by reverse transcription-quantitative polymerase chain reaction. Data are expressed as the mean \pm standard deviation. ${ }^{* *} \mathrm{P}<0.01$ vs. control group; ${ }^{\#} \mathrm{P}<0.05$ and ${ }^{\# \#} \mathrm{P}<0.01$, vs. model group. $\beta$-asarone $1 / 2 / 3$, depression model rats treated with $12.5,25$ or $50 \mathrm{mg} / \mathrm{kg} \beta$-asarone, respectively; FLU, fluoxetine-treated depression rats; CREB, cAMP response element binding protein; BDNF, brain-derived neurotrophic factor; Trk-B, tropomyosin receptor kinase B.

$\beta$-asarone enhances Bcl-2 level and reduces Bad in the hippocampus of CUMS-treated rats. The effect of $\beta$-asarone on the levels of Bcl-2 and Bad in the hippocampus of rats in the model group was also analyzed. As shown in Fig. 5, compared with the control group, the mRNA and protein levels of Bcl-2 were significantly decreased, while Bad levels were increased in the hippocampus of rats in the model group. However, (12.5, 25 and $50 \mathrm{mg} / \mathrm{kg} /$ day) $\beta$-asarone and $20 \mathrm{mg} / \mathrm{kg} /$ day fluoxetine treatment significantly eliminated these changes in Bcl-2 and Bad levels. $\beta$-asarone administered at $50 \mathrm{mg} / \mathrm{kg} /$ day exhibited the most marked effect on Bcl-2 and Bad levels in the hippocampus of CUMS-treated rats.

$\beta$-asarone increases ERK phosphorylation in the hippocampus of CUMS-treated rats. The ERK signaling pathway serves an important role by mediating the protective responses to stress (25). As shown in Fig. 6, CUMS decreased the phosphorylation of ERK in the hippocampus of rats, while $(12.5,25$ and $50 \mathrm{mg} / \mathrm{kg} /$ day $\beta$-asarone and $20 \mathrm{mg} / \mathrm{kg} /$ day fluoxetine treatment significantly increased the ERK phosphorylation as compared with that in the CUMS model group. $\beta$-asarone administered at $50 \mathrm{mg} / \mathrm{kg} /$ day had the most marked effect on p-ERK level in the hippocampus of CUMS-treated rats.

\section{Discussion}

Depression is a complex disorder that has become an important social problem due to the severe effect it has on the health and quality of life of patients (1). Thus far, drug treatment has been proven to be an effective method for depression treatment (26). Therefore, seeking novel and effective drugs for the treatment of depression is urgent.

$\beta$-asarone is the major active ingredient extracted from Acorus tatarinowii Schott. Acorus tatarinowii contains volatile oils, consisting mainly of $8.8-13.7 \% \alpha$-asarone and 63.2-81.2\% $\beta$-asarone, and has been used in traditional Chinese medicine formulas to relieve learning and memory deficits $(27,28)$. $\beta$-asarone can easily pass through the blood-brain barrier (7), and various studies have indicated that it has various pharmacological properties, including an anti-cancer, anti-inflammatory and neuroprotective effect $(12,29,30)$. In addition, $\beta$-asarone was able to relieve cognitive impairments in Alzheimer's disease (30), Parkinson's disease (31), and neuroinflammatory disease (32). Previous studies have also suggested the antidepressive effects of $\beta$-asarone (13-17). A study by Dong et al (33) suggested that $\beta$-asarone can reverse CUMS-induced depression-like behavior and induce hippocampal neurogenesis in rats. Nevertheless, to the best of our 
A

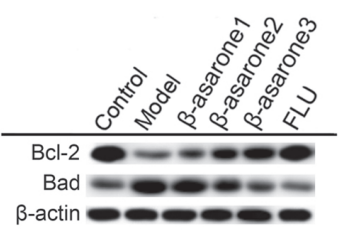

B

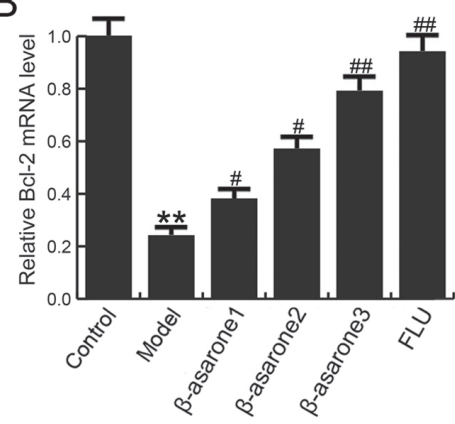

C

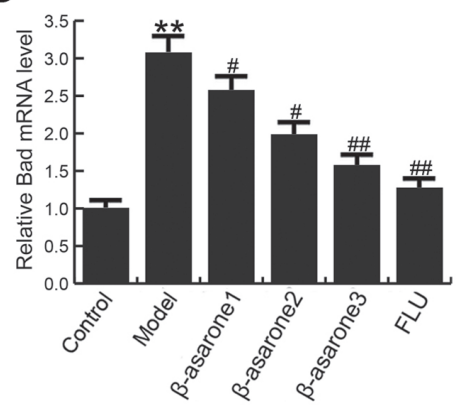

Figure 5. $\beta$-asarone enhances Bcl-2 level and reduces Bad level in the hippocampus of chronic unpredictable mild stress-treated rats. (A) Western blot analysis was conducted to examine Bcl-2 and Bad protein levels. (B) Bcl-2 and (C) Bad mRNA levels in different groups were detected by reverse transcription-quantitative polymerase chain reaction. Data are expressed as the mean \pm standard deviation. ${ }^{* *} \mathrm{P}<0.01$ vs. control group; ${ }^{\#} \mathrm{P}<0.05$ and ${ }^{\# \#} \mathrm{P}<0.01$, vs. model group. $\beta$-asarone1/2/3, depression model rats treated with $12.5,25$ or $50 \mathrm{mg} / \mathrm{kg} \beta$-asarone, respectively; FLU, fluoxetine-treated depression rats; Bcl-2, B-cell lymphoma-2; Bad, Bcl-2-associated death promoter.

A

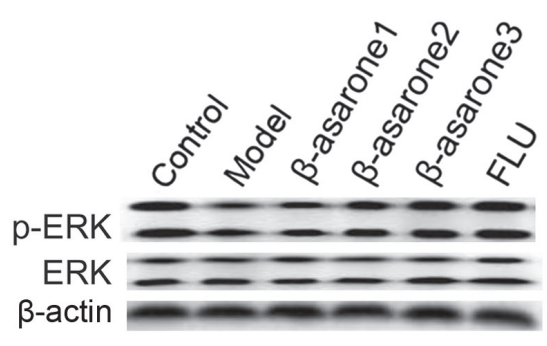

B

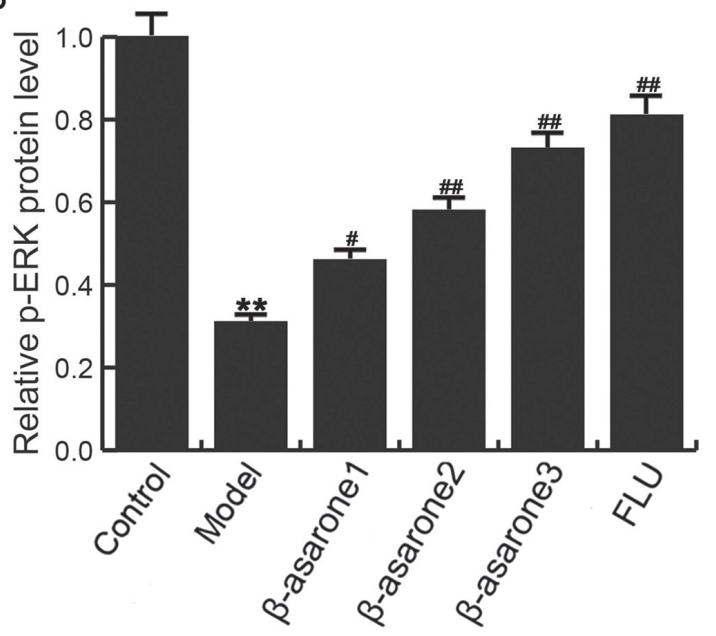

Figure 6. $\beta$-asarone increases ERK phosphorylation in the hippocampus of chronic unpredictable mild stress-treated rats. (A) Following treatment, the protein expression levels of ERK and p-ERK in different groups were detected by western blot analysis. (B) Quantified protein levels of p-ERK. Data were analyzed and expressed as the mean \pm standard deviation. ${ }^{* *} \mathrm{P}<0.01$ vs. control group; ${ }^{\#} \mathrm{P}<0.05$ and ${ }^{\# \#} \mathrm{P}<0.01$, vs. model group. $\beta$-asarone1/2/3, depression model rats treated with 12.5, 25 or $50 \mathrm{mg} / \mathrm{kg} \beta$-asarone, respectively; FLU, fluoxetine-treated depression rats; ERK, extracellular signal-regulated kinase; p-ERK, phosphorylated ERK.

knowledge, the molecular mechanisms underlying the effect of $\beta$-asarone in depression are not fully elucidated. Therefore, clarification of these mechanisms was attempted in the present study, and a rat model of depression induced by CUMS was established. Our investigation differed from the study of Dong et al (33) in various aspects: Firstly, in the current study, rats were subjected to different concentrations of $\beta$-asarone $(12.5,25$ and $50 \mathrm{mg} / \mathrm{kg}$ ), whereas the study by Dong et al only examined the dose of $25 \mathrm{mg} / \mathrm{kg} \beta$-asarone; secondly, fluoxetine $(20 \mathrm{mg} / \mathrm{kg})$ was used as the positive control drug in the present study, while this was not utilized in the previous study; and finally, the effect on the body weight and apoptosis in the hippocampus of depression rats was also explored in the present study. Consistent with the findings of the previous study (33), the current study also found that $\beta$-asarone significantly mitigated CUMS-induced depression-like behavior.

Hippocampal neuronal damage may be one of factors that trigger depression (1). Maggio and Segal (34) demonstrated that hippocampal neurons in a stress-depression rat model had a decreased proliferation ability, dendritic atrophy, increased apoptosis of pyramidal neurons and decreased hippocampal volume in rats with recurrent depression. The majority of current antidepressant drugs or methods exert their effect by promoting neuronal regeneration in the adult brain (35). Consistent with a previous study (23), the results of the present study indicated that CUMS treatment significantly induced hippocampal neuronal cell apoptosis, while $\beta$-asarone significantly decreased hippocampal neuronal cell apoptosis in CUMS rats.

BDNF, a recognized molecular marker of neuroplasticity, is one of the important members of the family of neurotrophic factors and is widely distributed in the central nervous system of mammals, particularly in the hippocampus, cortex and amygdala (36). Injection of BDNF in the brain to activate endogenous adult neural precursor cells can produce antidepressive effects (37). As one of the third messengers in the nucleus, CREB serves an important role in neurogenesis and neuroplasticity. CREB-mediated transcriptional alterations and central nervous system diseases are closely associated, 
such as cognitive decline, depression and cerebral ischemia (38). Activation of CREB in the hippocampal dentate gyrus of the adult rat hippocampus promotes neurogenesis, including cell proliferation and increased cell survival (39). At the same time, CREB also participates in the regulation of antidepressant therapy, and its activation mediates the change of neuronal plasticity induced by the antidepressant treatment. CREB achieves its function primarily through modulation of the target genes that are critical for the maintenance of synaptic function and cell survival. BDNF is one of the most important target genes of CREB and serves an important role in the pathological process of depression and antidepressant treatment. Furthermore, ERK1/2 is one of the mitogen-activated protein kinases involved in numerous cellular processes, including long-term neuronal plasticity and survival (40). Besides, the ERK signaling pathway is particularly important in mediating the protective responses to stress (25). ERK1/2 is upstream to CREB in the control of neurogenesis $(23,41)$. In the present study, the effect of $\beta$-asarone on the ERK1/2-CREB-BDNF pathway was further investigated. Consistent with a previous study (33), $\beta$-asarone was found to enhance p-ERK1/2, CREB, BDNF and Trk-B levels in the hippocampus of CUMS-treated rats. In addition, the anti-apoptotic gene $\mathrm{Bcl}-2$ and pro-apoptotic gene Bad, which serve critical roles in apoptosis regulation, were analyzed in the current study. It was observed that $\beta$-asarone enhanced Bcl-2 level and inhibited Bad level in the hippocampus of CUMS-treated rats. The data indicated that $\beta$-asarone activated the ERK1/2-CREB-BDNF pathway, enhanced $\mathrm{Bcl}-2$ expression and reduced $\mathrm{Bad}$ expression in the hippocampus of depression rats; however, the causal association remains unclear in vivo. This also requires in-depth investigation, thus, further research on this topic will be conducted in future studies.

In conclusion, the results of the present study indicated that $\beta$-asarone exerted an antidepressive effect on a rat model of depression induced by CUMS, and that the underlying molecular mechanism may be associated with the activation of the ERK signaling pathway. Therefore, $\beta$-asarone may be a novel and effective agent for the treatment of depression.

\section{Acknowledgements}

Not applicable.

\section{Funding}

The present study was supported by the Heilongjiang Provincial Education Department (grant no. 2016-KYYWF-0881).

\section{Availability of data and materials}

All datasets used and/or analyzed during the current study are available from the corresponding author on reasonable request.

\section{Authors' contributions}

HD and WC designed the current study and drafted the manuscript. HD, WC, XG, and YW collected the data and performed statistical analyses. ST, QL and CL performed statistical analysis and interpreted the data. All authors read and approved the final version of the manuscript.

\section{Ethics approval and consent to participate}

The present study was approved by the Ethics Committee of Qiqihar Medical University (Qiqihar, China).

\section{Patient consent for publication}

Not applicable.

\section{Competing interests}

The authors declare that they have no competing interests.

\section{References}

1. Alonso M, Vianna MR, Depino AM, Mello e Souza T, Pereira P, Szapiro G, Viola H, Pitossi F, Izquierdo I and Medina JH: BDNF-triggered events in the rat hippocampus are required for both short- and long-term memory formation. Hippocampus 12: 551-560, 2002.

2. Wu JL, Yu SY, Wu SH and Bao AM: A sensitive and practical RP-HPLC-FLD for determination of the low neuroactive amino acid levels in body fluids and its application in depression. Neurosci Lett 616: 32-37, 2016.

3. Kendler KS and Gardner CO: Dependent stressful life events and prior depressive episodes in the prediction of major depression: The problem of causal inference in psychiatric epidemiology. Arch Gen Psychiatry 67: 1120-1127, 2010.

4. Albert PR, Benkelfat C and Descarries L: The neurobiology of depression-revisiting the serotonin hypothesis. I. Cellular and molecular mechanisms. Philos Trans R Soc Lond B Biol Sci 367: 2378-2381, 2012.

5. Manji HK, Drevets WC and Charney DS: The cellular neurobiology of depression. Nat Med 7: 541-547, 2001.

6. Willner P, Towell A, Sampson D, Sophokleous S and Muscat R: Reduction of sucrose preference by chronic unpredictable mild stress, and its restoration by a tricyclic antidepressant. Psychopharmacology (Berl) 93: 358-364, 1987.

7. Fang YQ, Shi C, Liu L and Fang RM: Analysis of transformation and excretion of $\beta$-asarone in rabbits with GC-MS. Eur J Drug Metab Pharmacokinet 37: 187-190, 2012.

8. Zhang S, Xue ZF, Huang LP, Fang RM, He YP, Li L and Fang YQ: Dynamic expressions of Beclin 1 and tyrosine hydroxylase in different areas of 6-hydroxydopamine-induced Parkinsonian rats. Cell Mol Neurobiol 33: 973-981, 2013.

9. Liu L, Fang YQ, Xue ZF, He YP, Fang RM and Li L: Beta-asarone attenuates ischemia-reperfusion-induced autophagy in rat brains via modulating JNK, p-JNK, Bcl-2 and Beclin 1. Eur J Pharmacol 680: 34-40, 2012.

10. Geng Y, Li C, Liu J, Xing G, Zhou L, Dong M, Li X and Niu Y: Beta-asarone improves cognitive function by suppressing neuronal apoptosis in the beta-amyloid hippocampus injection rats. Biol Pharm Bull 33: 836-843, 2010.

11. Wang N, Zhang Q, Ning B, Luo L and Fang Y: $\beta$-Asarone promotes Temozolomide's entry into glioma cells and decreases the expression of P-glycoprotein and MDR1. Biomed Pharmacother 90: 368-374, 2017.

12. Wu J, Zhang XX, Sun QM, Chen M, Liu SL, Zhang X, Zhou JY and Zou X: $\beta$-Asarone inhibits gastric cancer cell proliferation. Oncol Rep 34: 3043-3050, 2015.

13. Liu DS, Xing GH, Lu CF, et al: Effect of $\beta$-asarone on the changes of specific enolase in hippocampus and serum neurons of CUMS rats. Chin J Gerontol 36: 1040-1041, 2016 (In Chinese).

14. Cai ZZ, Jin M, Yang PP, Li CC, Dong HY, Zhao AM and Zhang XJ; Qiqihaer Medical College: Effects of $\beta$-asarone on expression of endoplasmic reticulum stress regulators GRP78, ATF6 and XBP1 in hippocampus of depressive rats. Chin J New Drugs 26: 1053-1058, 2017. 
15. Zhao CM, Zhang XJ, Dong HY, et al: Effects of $\beta$-asarone on behavior and MKP-1, MSK-1, CREB and Bcl-2 in hippocampus of depressive rats. Chin J Exp Tradit Med Formulae 16: 272-277, 2013 (In Chinese)

16. Wang JP, Dong HY, Zhao CM, et al: Effects of $\beta$-asarone on behavior and biological clock related gene expression in depressive rats. Chin J Exp Tradit Med Formulae 2: 170-173, 2015 (In Chinese).

17. Dong HY, Zhang C, Wang JP, et al: Effects of $\beta$-asarone on the expression of circadian rhythm cycle gene Perl in depressive rats. Chin J New Drugs 7: 823-826, 2015 (In Chinese).

18. Deng XY, Li HY, Chen JJ, Li RP, Qu R, Fu Q and Ma SP: Thymol produces an antidepressant-like effect in a chronic unpredictable mild stress model of depression in mice. Behav Brain Res 291: 12-19, 2015.

19. Deng XY, Xue JS, Li HY, Ma ZQ, Fu Q, Qu R and Ma SP: Geraniol produces antidepressant-like effects in a chronic unpredictable mild stress mice model. Physiol Behav 152: 264-271, 2015.

20. Wang D, An SC and Zhang X: Prevention of chronic stress-induced depression-like behavior by inducible nitric oxide inhibitor. Neurosci Lett 433: 59-64, 2008.

21. Kurhe Y, Radhakrishnan M and Gupta D: Ondansetron attenuates depression co-morbid with obesity in obese mice subjected to chronic unpredictable mild stress; an approach using behavioral battery tests. Metab Brain Dis 29: 701-710, 2014

22. Li YC, Shen JD, Li J, Wang R, Jiao S and Yi LT: Chronic treatment with baicalin prevents the chronic mild stress-induced depressive-like behavior: Involving the inhibition of cyclooxygenase-2 in rat brain. Prog Neuropsychopharmacol Biol Psychiatry 40: 138-143, 2013

23. Li W, Zhu Y, Saud SM, Guo Q, Xi S, Jia B, Jiao S, Yang X, Lu J, Song S and Tu Y: Electroacupuncture relieves depression-like symptoms in rats exposed to chronic unpredictable mild stress by activating ERK signaling pathway. Neurosci Lett 642: 43-50, 2017.

24. Livak KJ and Schmittgen TD: Analysis of relative gene expression data using real-time quantitative PCR and the 2(-Delta Delta C(T)) method. Methods 25: 402-408, 2001.

25. Leem YH, Yoon SS, Kim YH and Jo SA: Disrupted MEK/ERK signaling in the medial orbital cortex and dorsal endopiriform nuclei of the prefrontal cortex in a chronic restraint stress mouse model of depression. Neurosci Lett 580: 163-168, 2014.

26. Cho H, Son SJ, Kim S and Park J: A randomized comparison of medication and cognitive behavioral therapy for treating depression in low-income young minority women. Med Sci Monit 22: 4947-4953, 2016

27. Rajput SB, Tonge MB and Karuppayil SM: An overview on traditional uses and pharmacological profile of Acorus calamus Linn. (Sweet flag) and other Acorus species. Phytomedicine 21: 268-276, 2014.

28. Kim JH, Hahm DH, Lee HJ, Pyun KH and Shim I: Acori graminei rhizoma ameliorated ibotenic acid-induced amnesia in rats. Evid Based Complement Alternat Med 6: 457-464, 2009.

29. Chang $W$ and Teng J: $\beta$-asarone prevents $A \beta 25$-35-induced inflammatory responses and autophagy in SH-SY5Y cells: Down expression Beclin-1, LC3B and up expression Bcl-2. Int J Clin Exp Med 8: 20658-20663, 2015.
30. Liu SJ, Yang C, Zhang Y, Su RY, Chen JL, Jiao MM, Chen HF, Zheng N, Luo S, Chen YB, et al: Neuroprotective effect of $\beta$-asarone against Alzheimer's disease: Regulation of synaptic plasticity by increased expression of SYP and GluR1. Drug Des Devel Ther 10: 1461-1469, 2016.

31. Zhang QS, Wang ZH, Zhang JL, Duan YL, Li GF and Zheng DL: Beta-asarone protects against MPTP-induced Parkinson's disease via regulating long non-coding RNA MALAT1 and inhibiting $\alpha$-synuclein protein expression. Biomed Pharmacother 83: 153-159, 2016.

32. Lim HW, Kumar H, Kim BW, More SV, Kim IW, Park JI, Park SY, Kim SK and Choi DK: $\beta$-Asarone (cis-2,4,5-trimethoxy-1-allyl phenyl), attenuates pro-inflammatory mediators by inhibiting NF- $\mathrm{KB}$ signaling and the JNK pathway in LPS activated BV-2 microglia cells. Food Chem Toxicol 72: 265-272, 2014.

33. Dong $\mathrm{H}$, Gao Z, Rong H, Jin M and Zhang X: $\beta$-asarone reverses chronic unpredictable mild stress-induced depression-like behavior and promotes hippocampal neurogenesis in rat. Molecules 19: 5634-5649, 2014.

34. Maggio N and Segal M: Differential modulation of long-term depression by acute stress in the rat dorsal and ventral hippocampus. J Neurosci 29: 8633-8638, 2009.

35. Piubelli C, Vighini M, Mathé AA, Domenici E and Carboni L: Escitalopram modulates neuron-remodelling proteins in a rat gene-environment interaction model of depression as revealed by proteomics. Part I: Genetic background. Int J Neuropsychopharmacol 14: 796-833, 2011.

36. You J, Yuan Y, Zhang Z, Zhang X, Li H and Qian Y: A preliminary association study between brain-derived neurotrophic factor (BDNF) haplotype and late-onset depression in mainland Chinese. J Affect Disord 120: 165-169, 2010.

37. Castrén E and Rantamäki T: The role of BDNF and its receptors in depression and antidepressant drug action: Reactivation of developmental plasticity. Dev Neurobiol 70: 289-297, 2010.

38. Ren X, Dwivedi Y, Mondal AC and Pandey GN: Cyclic-AMP response element binding protein (CREB) in the neutrophils of depressed patients. Psychiatry Res 185: 108-112, 2011.

39. Oury F, Yadav VK, Wang Y, Zhou B, Liu XS, Guo XE, Tecott LH, Schutz G, Means AR and Karsenty G: CREB mediates brain serotonin regulation of bone mass through its expression in ventromedial hypothalamic neurons. Genes Dev 24: 2330-2342, 2010.

40. Zhou X, Moon C, Zheng F, Luo Y, Soellner D, Nuñez JL and Wang H: N-methyl-D-aspartate-stimulated ERK1/2 signaling and the transcriptional up-regulation of plasticity-related genes are developmentally regulated following in vitro neuronal maturation. J Neurosci Res 87: 2632-2644, 2009.

41. Ha S and Redmond L: ERK mediates activity dependent neuronal complexity via sustained activity and CREB-mediated signaling. Dev Neurobiol 68: 1565-1579, 2008.

This work is licensed under a Creative Commons Attribution-NonCommercial-NoDerivatives 4.0 International (CC BY-NC-ND 4.0) License. 